\title{
COBERTURA DE COPAS COMO INDICADOR DE DESENVOLVIMENTO ESTRUTURAL DE REFLORESTAMENTOS DE RESTAURAÇÃO DE MATAS CILIARES NO MÉDIO VALE DO PARANAPANEMA, SP, BRASIL ${ }^{1}$
}

\author{
Antônio Carlos Galvão de $\mathrm{Melo}^{2}$, Dirceu Lúcio Carneiro de Miranda e Giselda Durigan²
}

\begin{abstract}
RESUMO - Muitos esforços e recursos têm sido despendidos com o objetivo de restaurar matas ciliares sem que tenham sido estabelecidos procedimentos para avaliação e monitoramento das áreas em restauração. Neste estudo, a cobertura de copas foi analisada como um indicador para avaliar os reflorestamentos de restauração. Em seis reflorestamentos de restauração com idades variando entre 1 e 3 anos, localizados no Médio Vale do Rio Paranapanema, São Paulo, Brasil, foram realizados levantamentos da estrutura da comunidade e estimada a cobertura pelo método da interseção na linha. A cobertura apresentou correlações significativas com idade $(\mathrm{r}=0,8140)$, altura $(\mathrm{r}=0,8002)$, área basal $(\mathrm{r}=0,7949)$, e diâmetro à altura do peito - DAP $(\mathrm{r}=0,7234)$ e foi considerada um indicador adequado para avaliar a evolução estrutural dos reflorestamentos, na faixa de idade enfocada neste estudo (até 36 meses). O modelo para determinação da taxa de cobertura de copas $(Y)$ esperada em função da idade $(X)$ dos plantios (meses) com melhor desempenho foi o modelo recíproco $(Y=a+b / X)$. Entretanto, outros modelos com melhores resultados de ajuste podem ser gerados caso seja considerado todo o espectro de variação de idades e de qualidade de sítio, que podem exercer influência na evolução da cobertura.
\end{abstract}

Palavras-chave: Indicadores, matas ciliares e restauração.

\section{CROWN COVER AS INDICATOR OF STRUCTURAL DEVELOPMENT OF RIPARIAN AREAS RESTORATION IN THE MID-PARANAPANEMA VALLEY, BRAZIL}

\begin{abstract}
Much inspection efforts and funds have been expended with the aim of restoring riparian forests, in spite of the absence of clearly defined procedures to assess the reforested areas. In this study, canopy cover is analyzed as an indicator to be easily and precisely applied to evaluate the efficacy in forest restoration. Six reforested areas ranging from one to three years old, located at the Mid-Paranapanema Valey, São Paulo State, Brazil, were included in the structural analysis that supported this study. Crown cover estimated by line interception showed significant correlations with age $(r=8140)$; height $(r=8002)$; basal area $(r=$ $7949)$ and diameter at breast height - DBH $(r=7234)$ and was considered a suitable indicator to assess the structural evolution of planted riparian forests at the ages comprised by this study (up to 36 months). The best equation for estimating crown cover rate by line interception $(Y)$ as a function of age $(X)$ was determined $(Y=a+b / X)$. Better equations can be obtained if the whole range of ages and site variation are considered.
\end{abstract}

Keywords: Indicators, restoration and riparian forests.

\footnotetext{
${ }^{1}$ Recebido em 07.03.2006 e aceito para publicação em 08.02.2007.

${ }^{2}$ Instituto Florestal, Secretaria de Meio Ambiente do Estado de São Paulo, Floresta Estadual de Assis, Cx.P.104, 19800-000 Assis, Sp. E-mail: <acgmelo@gmail.com>.

${ }^{3}$ Departamento de Ciências Florestais da Universidade Federal do Paraná. Av.Lothário Meissner, 3400, 80210-170 Curitiba, PR.
} 


\section{INTRODUÇÃO}

O monitoramento da qualidade ambiental é fundamental para a avaliação da efetividade dos esforços aplicados em sua conservação, e os métodos de avaliação e monitoramento de processos ambientais têm se pautado pela utilização de indicadores que são parâmetros que permitem avaliar atributos de áreas ou processos, possibilitando monitorar tendências de mudanças ambientais (DAJOZ, 1973) ou diagnosticar causas de um problema ambiental (DALE e BEYELER, 2001).

O desenvolvimento de indicadores é uma área crítica da pesquisa em ecologia florestal, pois, ainda que a fundamentação para a sua seleção esteja razoavelmente bem estabelecida, a maior tarefa remanescente é testálos e validá-los (NOSS, 1999). Para Eiswert e Haney (2001), a confiabilidade dos indicadores depende de atributos como sensibilidade, especificidade e capacidade de predição.

A seleção de indicadores deve, segundo Manoliadis (2002):

i) Estar intimamente ligada aos objetivos do projeto.

ii) Propiciar a abordagem efetiva do processo.

iii) Ser definida claramente, de forma a evitar confusões na aplicação ou interpretação.

iv) Ser realista e considerar os custos para sua coleta.

v) Ter alta qualidade e confiabilidade.

vi) Considerar escalas temporal e espacial adequadas.

No Brasil, a partir da década de 1980 o desenvolvimento de técnicas de restauração de matas ciliares tornou-se mais intenso, bem como a edição de normas legais sobre o assunto e a aplicação de recursos públicos e privados em projetos executivos. Apesar disso, só recentemente o monitoramento de áreas em restauração tem sido objeto de pesquisas. A seleção de indicadores foi discutida, dentre outros, por Rodrigues e Gandolfi (1998, 2000), Mantovani (1998) e Almeida e Sánchez (2005).

Nos casos dos processos de restauração de matas ciliares, há que se considerar alguns aspectos que devem ser norteadores na escolha dos indicadores:

- A grande demanda para avaliação dos projetos de restauração de matas ciliares parte dos órgãos de financiamento, fomento e fiscalização, que devem monitorar os projetos durante dois ou, no máximo, três anos após a sua implantação (BARBOSA, 2001).

- Os projetos de restauração devem contemplar medidas que propiciem o retorno dos processos ecológicos originais e, para isso, têm de ser adotadas medidas que possibilitem o fim das perturbações, a criação de condições para estabelecimento de propágulos (sementes ou mudas), o controle de organismos prejudiciais e a sustentabilidade do processo, entendida como a capacidade da área restaurada de se perpetuar (MANTOVANI, 1998; RODRIGUES e GANDOLFI, 2000).

Dentre os parâmetros estruturais dos reflorestamentos de restauração, merece especial destaque a cobertura de copas, definida por GreigSmith (1983) como "a proporção do solo ocupada pela projeção perpendicular da parte aérea dos indivíduos da população ou comunidade em análise".

A cobertura promovida pelo dossel controla a quantidade, qualidade e distribuição temporal e espacial da luz, determinando níveis diferenciados de umidade do ar, temperatura e condições de umidade do solo (JENNINGS et al., 1999), além de promover a interceptação das chuvas, reduzindo o impacto direto sobre o solo. Dessa forma, a cobertura é o maior determinante do "micro-habitat" interno da floresta, afetando o crescimento e sobrevivência de plântulas, determinando a composição florística da comunidade, afetando processos de oxidação da matéria orgânica e controlando processos erosivos.

O rápido incremento da cobertura é importante nos processos de restauração, pois a diminuição da luminosidade incidente no solo é fator importante para o controle da mato-competição (GUILHERME, 2000; MARTINS et al., 2004).

Várias são as técnicas para estimativa da cobertura, que acabam se dividindo em três categorias, baseadas em diferentes unidades geométricas: plano, ponto e linhas (FLOYD e ANDERSON, 1987).

Métodos baseados no plano utilizam a estimativa da cobertura de copas por meio do cálculo do somatório das áreas de projeção das copas em relação à área de amostragem, que é conhecido como método de parcelas ou dos diâmetros de copa (MÜELLER-DOMBOIS e ELLEMBERG, 1974). Tal método depende da demarcação das parcelas em campo, além da mensuração das 
projeções de copas individualmente, o que acaba por demandar maior tempo para levantamento dos dados e dos cálculos posteriores, além da imprecisão devida à variação do formato das copas.

Os métodos baseados na interseção de pontos prevêem a contagem do número de interseções pelas copas que ocorrem nas projeções verticais de cada um dos pontos estabelecidos para dada área a ser amostrada. A cobertura é estimada a partir do cálculo da proporção de pontos interceptados (GREIG-SMITH, 1983). Tais métodos são influenciados pela deciduidade, sendo necessária, para uma boa caracterização da comunidade, a realização de pelo menos duas medidas anuais. São, também, de difícil medição para comunidades arbóreas. Esse método foi utilizado por Cunha et al. (2003) para a avaliação de processos de restauração de dunas.

O método de cálculo por meio da interseção de linhas, inicialmente descrito por Canfield (1941), considera como cobertura o somatório de interseções das projeções de copas sobre uma linha, dividido pelo comprimento total dessa linha, expresso em porcentagem.

Floyd e Anderson (1987), comparando três métodos de estimativa de cobertura, concluíram que, para se atingirem graus de precisão semelhantes, em comunidades que incluam outras formas de vida além da arbustiva, o método da projeção de copas demanda maior tempo de aplicação do que os de interseção de pontos e o de interseção na linha. O de interseção de pontos demanda cerca de dois terços do tempo de aplicação daquele observado para o de interseção na linha. Além da maior demanda de tempo, Mueller-Dombois e Ellemberg (1974) afirmaram que o método de projeção de copas não é prático quando se pretende estimar a cobertura em áreas grandes.

Jennings et al. (1999), estudando diferentes métodos para estimar cobertura, concluíram que não é possível recomendar "o melhor método", posto que a decisão por um deles depende da natureza do estudo. Esses autores ressaltaram que limitações logísticas e operacionais seriam muito importantes na decisão sobre qual método utilizar.

O método de interseção de linhas foi empregado por Meeuwig e Budy (1981) para a estimativa de biomassa e taxas de crescimento e a determinação de modelos de crescimento para diversos parâmetros estruturais em florestas temperadas. No Brasil, Durigan et al. (2002) aplicaram esse método para caracterização de vegetação de Cerrado no Estado de São Paulo, enquanto Meirelles et al. (2002) utilizaram-no para levantamento do estrato herbáceo do Cerrado no Maranhão.

O presente estudo teve por objetivo avaliar a cobertura de copas pelo método de interseção de linhas, como indicador estrutural do desenvolvimento de reflorestamentos de restauração de matas ciliares em plantios jovens, com idade até 36 meses.

\section{MATERIAL E MÉTODOS}

As áreas selecionadas para este estudo estão localizadas no médio Vale do Paranapanema, sudoeste do Estado de São Paulo, Brasil (Tabela 1). Nesta região os solos são profundos, argilosos e férteis, em altitudes que variam de 330 a 440 metros acima do nível do mar, com predominância do uso para agricultura de grãos e de cana-de-açúcar. O clima é caracterizado como Cfa (classificação de Köppen), com precipitação média de $1.350 \mathrm{~mm}$ (SILVEIRA e DURIGAN, 2004). A vegetação original da região é classificada, segundo o sistema de Veloso et al. (1991), como Floresta Estacional Semidecidual, um dos subtipos da Mata Atlântica.

Foram selecionados seis reflorestamentos que tivessem históricos similares de ocupação anterior à restauração, que se localizassem sobre solos similares, em fertilidade e umidade, e que tivessem sido submetidos a um conjunto de operações semelhantes de plantio e manutenção. Todos eles foram executados com base em modelo sucessional, utilizando-se de, no mínimo, $50 \%$ de árvores de espécies pioneiras da região, como Citharexylum myrianthum Cham, Croton floribundus (L.) Spreng, Croton urucurana Baill. e Trema micrantha (L.) Blume. Na Tabela 1 é apresentada uma breve descrição das características de cada uma das áreas deste estudo.

Para o levantamento das características estruturais dos reflorestamentos foram instaladas, em cada área de estudo, cinco parcelas de $100 \mathrm{~m}^{2}(10 \mathrm{~m} \times 10 \mathrm{~m})$. Todas as árvores plantadas no interior das parcelas foram registradas, e, para as plantas maiores que $1,7 \mathrm{~m}$ de altura, foram medidos o diâmetro à altura do peito (DAP) com suta e a altura total com régua dendrométrica telescópica.

As linhas para estimativa da cobertura pelo método da interseção foram locadas de forma oblíqua às linhas de plantio, e cada uma delas foi sobreposta diagonalmente

R. Árvore, Viçosa-MG, v.31, n.2, p.321-328, 2007 
a uma parcela de levantamento estrutural dos reflorestamentos. Adotaram-se 15 m como extensão total de cada amostra, por terem os reflorestamentos de restauração estrutura intermediária entre florestas adultas e densas - para as quais se recomendam 20 m de extensão(MEEUWIGe BUDY, 1981)-e comunidades de arbustos para as quais se recomendam entre $11 \mathrm{e}$ 13 m (FLOYD e ANDERSON, 1987).

Para investigar a associação da cobertura obtida através do método de interseção de linhas com outras variáveis da estrutura e idade dos reflorestamentos foi construída, com dados individualizados por parcela, a matriz de correlações Spearman.

Os valores de cobertura obtidos pelo método de interseção em linha foram submetidos ao teste de intensidade amostral, fixados no nível de probabilidade de $95 \%$ e com erro admitido de $10 \%$, com a finalidade de traçar recomendações acerca dos procedimentos de amostragem para tal técnica.
Foram desenvolvidos modelos para a estimativa da cobertura dos reflorestamentos de restauração em função de sua idade, visando avaliar a pertinência de se predizerem níveis ideais de desenvolvimento dos reflorestamentos para diferentes idades. Para tanto e na falta de modelos já desenvolvidos com essa finalidade, foram testados modelos gerados pelo software Statgraphics Plus 4.1. Os critérios utilizados para a escolha do melhor modelo foram a análise do coeficiente de determinação $\left(\mathrm{R}^{2}\right)$, o erro-padrão da estimativa (Syx\%) e a distribuição gráfica dos resíduos.

\section{RESULTADOS E DISCUSSÃO}

Os valores das variáveis estruturais dos reflorestamentos indicaram que ocorre incremento dos parâmetros estruturais com o aumento da idade (Tabela 2), conforme esperado de reflorestamentos fundamentados na sucessão secundária (RODRIGUES e GANDOLFI, 2000).

Tabela 1 - Localização, idade, área, solos, ocupação anterior, densidade de plantio e riqueza de espécies de seis reflorestamentos de restauração de matas ciliares selecionados para estudos e estabelecimento de parâmetros de avaliação da restauração na região do Médio Paranapanema, São Paulo, Brasil

Table 1 - Location, restoration age, area, soil, planting density and species richness of six riparian reforestation sites selected for restoration assessment in the Mid-Paranapanema Valley, São Paulo, Brazil

\begin{tabular}{|c|c|c|c|c|c|}
\hline Área & Coordenadas & $\begin{array}{c}\text { Idade } \\
\text { (meses) }\end{array}$ & $\begin{array}{l}\text { Área } \\
\text { (ha) }\end{array}$ & Solo $^{(1)}$ & Ocupação anterior \\
\hline 1 & $\begin{array}{c}22^{\circ} 47^{\prime} 58^{\prime \prime} \mathrm{S} \\
50^{\circ} 37^{\prime} 35^{\prime}, \mathrm{W}\end{array}$ & 16 & 0,50 & $\begin{array}{l}\text { Associação Latossolo Roxo + Terra Roxa } \\
\text { Estruturada Latossólica, ambos Eutróficos }\end{array}$ & $\begin{array}{l}\text { Agricultura (pelo } \\
\text { menos } 25 \text { anos) }\end{array}$ \\
\hline 2 & $\begin{array}{l}22^{\circ} 48^{\prime} 42^{\prime \prime} \mathrm{S} \\
50^{\circ} 33^{\prime} 08^{\prime}, \mathrm{W}\end{array}$ & 13 & 4,00 & $\begin{array}{l}\text { Associação Latossolo Roxo + Terra Roxa } \\
\text { Estruturada Latossólica, ambos Eutróficos }\end{array}$ & $\begin{array}{l}\text { Agricultura (pelo } \\
\text { menos } 20 \text { anos) }\end{array}$ \\
\hline 3 & $\begin{array}{l}22^{\circ} 41^{\prime} 55^{\prime}, \mathrm{S} \\
50^{\circ} 50^{\prime} 52^{\prime}, \mathrm{W}\end{array}$ & 13 & 3,80 & Latossolo roxo Eutrófico & $\begin{array}{l}\text { Agricultura (pelo } \\
\text { menos } 40 \text { anos) }\end{array}$ \\
\hline 4 & $\begin{array}{l}22^{\circ} 48^{\prime} 28^{\prime}, \mathrm{S} \\
50^{\circ} 50^{\prime} 45^{\prime}, \mathrm{W}\end{array}$ & 36 & 0,76 & Latossolo Vermelho Escuro Eutrófico & $\begin{array}{l}\text { Pastagem (pelo } \\
\text { menos } 33 \text { anos) }\end{array}$ \\
\hline 5 & $\begin{array}{l}22^{\circ} 46^{\prime} 42^{\prime \prime} \mathrm{S} \\
50^{\circ} 35^{\prime} 22^{\prime}, \mathrm{W}\end{array}$ & 36 & 0,63 & $\begin{array}{l}\text { Associação Latossolo Roxo + Terra Roxa } \\
\text { Estruturada Latossólica, ambos Eutróficos }\end{array}$ & $\begin{array}{l}\text { Agricultura (pelo } \\
\text { menos } 25 \text { anos) }\end{array}$ \\
\hline 6 & $\begin{array}{l}22^{\circ} 43^{\prime} 32^{\prime \prime} \mathrm{S} \\
50^{\circ} 33^{\prime} 37^{\prime}, \mathrm{W}\end{array}$ & 36 & 2,31 & $\begin{array}{l}\text { Associação Latossolo Roxo + Terra Roxa } \\
\text { Estruturada Latossólica, ambos Eutróficos }\end{array}$ & $\begin{array}{l}\text { Agricultura (pelo } \\
\text { menos } 20 \text { anos) }\end{array}$ \\
\hline
\end{tabular}

Fontes: (1) Bognola et al. (1990)

Tabela 2 - Riqueza, densidade, área basal, altura média e cobertura de copas do estrato arbóreo de seis reflorestamentos de restauração ripária no Médio Vale do Paranapanema, São Paulo, Brasil

Table 2 - Species richness, density, basal area, medium height and crown cover of six planted riparian forests in the MidParanapanema Valley, São Paulo, Brazil

\begin{tabular}{lccccc}
\hline & Área 1 & Área 2 & Área 3 & Área 4 & Área 5 \\
\hline Riqueza de espécies & 32 & 11 & 26 & 41 & 12 \\
Densidade (árvores.ha. $\left.{ }^{-1}\right)$ & 1320 & 1240 & 1320 & 2200 & 1240 \\
Área basal $\left(\mathrm{m}^{2} / \mathrm{ha}\right)$ & 4,39 & 0,52 & 1,77 & 16,23 & 1580 \\
Altura média (m) & 2,98 & 1,55 & 2,26 & 5,26 & 15,36 \\
Cobertura de copas $(\%)$ & 108,13 & 19,27 & 44,67 & 179,67 & 6,07 \\
\hline
\end{tabular}

R. Árvore, Viçosa-MG, v.31, n.2, p.321-328, 2007 
Os resultados de altura dos plantios de três anos foram superiores àqueles encontrados por Almeida e Sánchez (2005) em áreas de restauração com 5 e 7 anos no Vale do Paraíba, SP. Entretanto, não existem na literatura informações para plantios de mesma idade na mesma região, o que torna impossível a comparação dos dados. Diferenças de valores observadas entre plantios de mesma idade podem ser atribuídas à composição de espécies utilizadas nos plantios ou a diferenças de qualidade do sítio, que só poderiam ser detectadas por meio de estudos complementares, principalmente com relação aos solos.

As correlações encontradas entre a cobertura obtida pela interseção na linha e as demais variáveis estruturais dos reflorestamentos foram expressivas para: idade, altura média, DAP médio e área basal (Tabela 3). Esses resultados dão suporte à recomendação de se utilizar a cobertura obtida pelo método da interseção na linha como um bom indicador da evolução estrutural dos reflorestamentos de restauração para plantios com idade de até 36 meses.

A intensidade amostral ideal para a estimativa da cobertura de copas pelo método da interseção, calculada com base na amostragem realizada em cada um dos reflorestamentos analisados, variou, nos plantios de um ano, entre 20 e 65 linhas de 15 metros e entre 9 e 19 linhas para os plantios de 3 anos.

Canfield (1941) previu, para o método da interseção na linha, a necessidade de adequação da amostragem com a heterogeneidade da vegetação em análise e sugeriu um número mínimo de 16 linhas. Floyd e Anderson (1987), estudando a cobertura de arbustos em área de estepe nos Estados Unidos, sugeriram 20 linhas de 11 metros para a espécie Sitanion hystrix, 28 linhas de 13 metros para Oryzopis hymenoides e 20 linhas de 12 metros para ervas, em geral.

Durigan et al. (2002) e Meirelles et al. (2002) utilizaram o método da interseção na linha para levantamentos de vegetação de Cerrado, mas nenhum dos trabalhos apresenta conclusões acerca da intensidade amostral desejável.

Considerando a literatura disponível sobre o assunto e a grande variabilidade dos reflorestamentos de restauração que são implantados com grande número de espécies de diferentes desempenhos em crescimento, é razoável propor que a estimativa de cobertura de reflorestamentos de restauração seja iniciada utilizandose 10 linhas de 15 metros. Cálculos de intensidade amostral de cada área sob monitoramento devem ser realizados e indicarão, em cada caso, a necessidade de complementação da amostragem.

Os modelos gerados para predição da cobertura de copas em função da idade são apresentados na Tabela 4. O modelo recíproco, de número 1 , foi considerado mais adequado, pois apresentou o maior coeficiente de determinação $\left(R^{2}=0,722\right)$, menor erro-padrão da estimativa $($ Syx \% $=31,8 \%)$ e a menor dispersão dos resíduos (Figura 1).

Tabela 3 - Correlações de Pearson entre idade, densidade, altura média, diâmetro médio à altura do peito médio, área basal e cobertura do estrato arbóreo de seis reflorestamentos de restauração, com idades de 1 e 3 anos, no Médio Vale do Paranapanema, São Paulo, Brasil (DAP = diâmetro à altura do peito)

Table 3 - Pearson's correlations among age, density, medium height, medium diameter at breast height, basal area and crown cover of arboreal layer for six riparian forest restoration plantings with ages ranging from one to three years, in the Mid-Paranapanema Valley, São Paulo, Brazil (DAP = diameter at breast height)

\begin{tabular}{|c|c|c|c|c|c|c|}
\hline & Idade & Densidade & $\begin{array}{l}\text { Altura } \\
\text { média }\end{array}$ & $\begin{array}{l}\text { DAP } \\
\text { médio }\end{array}$ & $\begin{array}{l}\text { Área } \\
\text { Basal }\end{array}$ & $\begin{array}{c}\text { Cobertura } \\
\text { de copas }\end{array}$ \\
\hline$\overline{\text { Idade }}$ & 1,000 & & & & & \\
\hline Densidade & $0,482 *$ & 1,000 & & & & \\
\hline Altura média & $0,919^{*}$ & 0,397 & 1,000 & & & \\
\hline DAP médio & $0,874 *$ & 0,239 & $0,912 *$ & 1,000 & & \\
\hline Área Basal & $0,908^{*}$ & $0,470 *$ & $0,843^{*}$ & $0,903 *$ & 1,000 & \\
\hline Cobertura de copas & $0,814 *$ & $0,586 *$ & $0,800 *$ & $0,723 *$ & $0,795^{*}$ & 1,000 \\
\hline
\end{tabular}

$*$ correlação significativa a $\rho<0,001 / \rho<0,001$ significant 
Tabela 4 - Equações para predição de cobertura em função da idade de reflorestamentos de restauração de matas ciliares com idades variando de 1 a 3 anos de idade na região do Medio Paranapanema, SP, Brasil ( $Y=$ cobertura em porcentagem, $X=$ idade em meses, $\mathrm{n}=30$ e $\mathrm{GL}=28$ )

Table 4 - Equations for crown cover prediction for riparian forest restoration plantings with ages ranging from one to three years, in the Mid-aranapanema Valley, Brazil ( $Y=$ crown cover \%; X=age in months)

\begin{tabular}{cccccc}
\hline & Modelo & $a$ & $b$ & $\mathrm{R}^{2}$ & Syx\% \\
\hline 1 & $Y=a+b / X$ & 219,668 & $-2294,92$ & 0,722 & 31,8 \\
2 & $Y=a+b X$ & $-7,80984$ & 4,51418 & 0,662 & 35,1 \\
3 & $Y=a+b * \log (X)$ & $-224,982$ & 106,158 & 0,691 & $\mathrm{p}<0.00001$ \\
\hline
\end{tabular}

a) Modelo 1

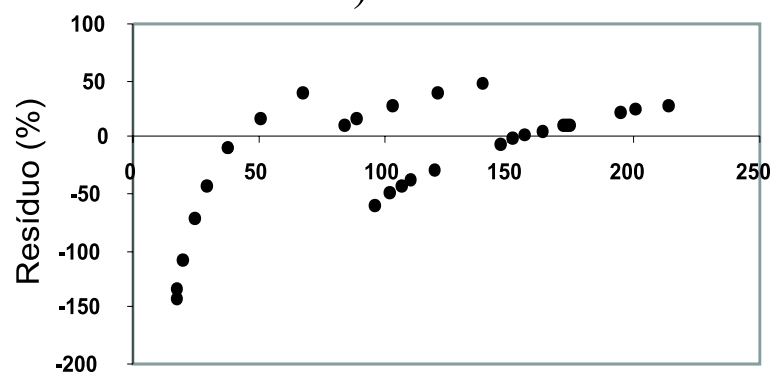

b) Modelo 2

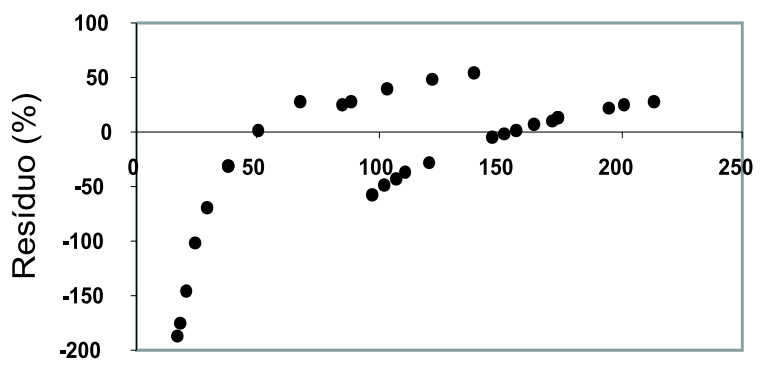

c) Modelo 3

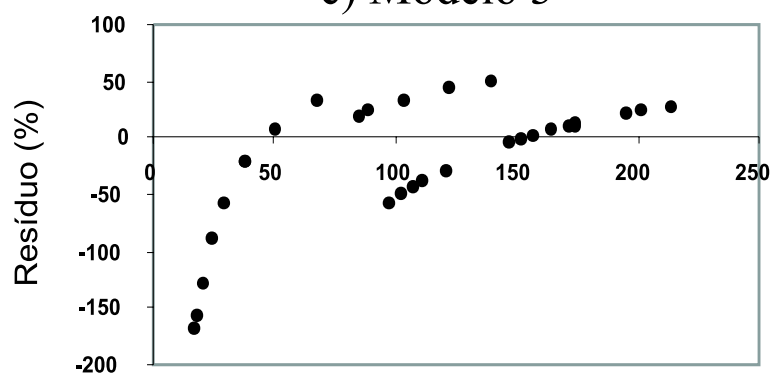

Figura 1 - Distribuição gráfica dos resíduos porcentuais em três diferentes modelos ajustados para estimativa de cobertura de copas em função da idade em reflorestamentos de restauração de matas ciliares.

Figure 1 -Residual graphical analysis for three crown cover estimative equations as a function of age of planted trees.
Não foram encontradas na literatura referências que tratassem da modelagem com os mesmos propósitos deste estudo. São mais comuns estudos que desenvolvem ou ajustam modelos alométricos com base em parâmetros dendrométricos (altura, DAP ou área transversal) como variáveis independentes, para a predição da área de copas de cada árvore (MEEUWIG e BUDY, 1981; GILL et al., 2000) ou de forma inversa, utilizando cobertura de copas para predizer características da comunidade, como um todo (TAUSCH e TUELLER, 1977; KNOWLES et al., 2004; DONOSO, 2005).

Os modelos testados indicaramm uma boa correlação entre a cobertura de copas e a idade do reflorestamento. Entretanto, mesmo para o melhor modelo, as medidas de precisão ( $\mathrm{R}^{2}$ e Syx\%) não permitem recomendar sua utilização irrestrita. A grande variação observada entre as áreas amostradas, que pode ter origem na qualidade do sítio, na composição de espécies adotada ou na lacuna de amostragem nas idades entre 16 e 36 meses, indica que o desenvolvimento de modelos carece de maior intensidade amostral, abrangendo maior número de áreas e, principalmente, incluindo reflorestamentos com idades não analisadas neste estudo.

\section{CONCLUSÕES}

A cobertura de copas estimada pelo método de interseção de linhas é um indicador adequado para avaliação dos reflorestamentos de restauração, e sua estimativa deve ser acompanhada de avaliação da intensidade amostral.

Modelos para predição da cobertura de copas em função da idade do reflorestamento de restauração podem ser desenvolvidos, desde que consideradas as variações de idade e qualidade de sítio que podem exercer infuência no desempenho de evolução da cobertura. 


\section{AGRADECIMENTOS}

À Fundação de Amparo à Pesquisa do Estado de São Paulo - FAPESP; pelo financiamento parcial deste estudo (Processo FAPESP 00/02020-9).

\section{REFERÊNCIAS}

ALMEIDA, R.O.P.O.; SÁNCHEZ, L.E. Revegetação de áreas de mineração: critérios de monitoramento e avaliação de desempenho. Revista Árvore, v.29, n.1, p.47-54, 2005.

BARBOSA, L. M. (Coord.). Modelos de repovoamento vegetal para proteção de recursos hídricos em áreas degradadas dos diversos biomas no Estado de São Paulo. São Paulo. São Paulo: Instituto de Botânica, 2001. 185p. (Relatório de atividades - Fase I - Processo FAPESP 00/02020-9).

BOGNOLA, I. A. et al. Levantamento pedológico semidetalhado da região de governo de Assis. Escala 1:50.000. Convênio IAC/CIERGA/IGC, 1990.

CANFIELD, R.H. Application of the line interception method in sampling range vegetation. Journal of Forestry, v.39, p.388-394, 1941.

CUNHA, L.O. et al. Análise multivariada da vegetação como ferramenta para avaliar a reabilitação de dunas litorâneas mineradas em Mataraca, Paraíba, Brasil. Revista Árvore, v.27, n.4, p.503-515, 2003.

DAJOZ, R. Ecologia geral. Petrópolis: Vozes, 1973. 472p.

DALE, V.H.; BEYELER, S.C. Challenges in the development and use of ecological indicators. Ecological Indicators, v.1, p.3-10, 2001.

DONOSO, J. Crown index: a cannopy balance indicator to assess growth and regeneration in uneven-aged stands of Coastal Range of Chile. Forestry, v.78, n.4, p.337-351, 2005.

DURIGAN, G. et al. Caracerização de dois estratos da vegetação em uma área de cerrado no município de Nrotas, SP, Brasil. Acta Botânica Brasílica, v.16, n.3, p.251-262, 2002.
EISWERT, M.E.; HANEY, J.C. Maximizing

conserved biodiversity: why ecosystem indicators and tresholds matter. Ecological Economics, v.38, p.259-274, 2001.

FLOYD, D.A.; ANDERSON, J.E. A Comparison of three methods for estimating plant cover. Journal of Ecology, v.75, p.221-228, 1987.

GILL, S.J.; BIGING, G.S.; MURPHY, E.C. Modeling conifer tree crown radius and estimating canopy cover. Forest Ecology and Management, v.126, n.3, p.405-416, 2000.

GREIG-SMITH, P. Quantitative plant

ecology. 2 ed. London: Butterworths, 1983. 256p.

GUILHERME, F.A.G. Efeitos da cobertura de dossel na densidade e estatura de gramíneas e da regeneração natural de plantas lenhosas em mata de galeria, Brasília, DF. Cerne, v.6, n. 1, p.60-66, 2000.

JENNINGS, S.B.; BROWN, N.D.; SHEIL, D. Assessing forest canopies and understorey illumination: canopy closure, canopy cover and oyther measures. Forestry, v.72, n.1, p.59-73, 1999.

KNOWLES, R.L. et al. Developing a canopy closure model to predict overstory / understory relationships in Pinus radiate silvopastoral systems. Agroforestry Systems, v.43, n.1-3, p.109-119, 2004.

MANOLIADIS, O.G. Development of ecological indicators - a metodologia framework usina comprimisse programming. Ecological Indicators, v.2, p.169-176, 2002.

MANTOVANI, W. Recuperação e monitoramento de ecossistemas: escalas de abordagem. In: SIMPOSIO DE ECOSSISTEMAS BRASILEIROS, 4., 1998. Anais... Águas de Lindóia: 1998. v.5, p.228294. (Publicações ACIESP1, 104).

MARTINS, C.R.; LEITE, L.L.; HARIDASAN, M. Capim-gordura (Melinis minutiflora $\mathrm{P}$. Beauv.), uma gramínea exótica que compromete a recuperação de áreas degradadas em unidades de conservação. Revista Árvore, v. 28, n.5, p.739-747, 2004.

R. Árvore, Viçosa-MG, v.31, n.2, p.321-328, 2007 
MEeUWIG, R.O.; BUDY, J.D. Point and lineintercect sampling in pinyon-juniper woodlands. Washington: USDA Forest Service, 1981. 38p. (General Technical Report INT 104).

MEIRELLES, M.L. et al. Utilização do método de interseção na linha em levantamento quantitativo do estrato herbáceo do cerrado. Boletim de Herbicidas Ezechias Heringer, v.9, p.60-68, 2002.

MUELLER-DOMBOIS, D.; ELLEMBERG, H. Aims and methods of vegetation ecology. New York: Wiley \& Sons, 1974. 547p.

NOSS, R.F. Assessing and monitoring forest biodivesity: a suggested framework and indicators. Forest Ecology and Management, v.115, p.135-146, 1999.

RODRIGUES, R.R.; GANDOLFI, S. Restauração de florestas tropicais: subsídios para uma definição metodológica e indicadores de avaliação e monitoramento. In: DIAS, L. E.; MELO, J. W. V. Recuperação de Áreas Degradadas. Viçosa, MG: Universidade Federal de Viçosa; Sociedade Brasileira de Recuperação de Áreas Degradadas, 1998. p.203-215.
RODRIGUES, R.R.; GANDOLFI, S. Conceitos, tendências e ações para a recuperação de formações ciliares In: RODRIGUES, R.R.; LEIT ÃO FILHO, H.F. (Ed.). Matas ciliares: conservação e recuperação. São Paulo: Universidade de São Paulo/Fapesp, 2000. p.235-247.

SILVEIRA, E.R..; DURIGAN, G. Recuperação da matas ciliares: estrutura da floresta e regeneração natural aos 10 anos em diferentes modelos de plantio na Fazenda Canaçu, Tarumã, S.P. In: VILAS BOAS, O.; DURIGAN, G.

Pesquisas em conservação e recuperação ambiental no Oeste Paulista: resultados da cooperação Brasil/Japão. São Paulo: Páginas e Letras, 2004. p.347-370.

TAUSCH, R.J.; TUELLER, P.T. Plant succession following chaining of pinyon-juniper woodlands in eastern Nevada. Journal of Range Management, v.30, n.1, p.44-49, 1977.

VELOSO, H.P.; RANGEL FILHO, A.L.; LIMA, J.C.A. Classificação da vegetação brasileira adaptada a um sistema universal. Rio de Janeiro: IBGE, 1991. 123p. 